\title{
Mismatched unrelated donor allogeneic stem cell transplant for high risk haematological malignancy: A single centre experience
}

\author{
Heshani Mediwake ${ }^{1,2}$, Cameron Curley ${ }^{1}$, Jason Butler ${ }^{1}$, Angela Mclean ${ }^{1}$, Siok Tey ${ }^{1,3}$, Geoffrey R. Hill ${ }^{1,3}$, Anthony Morton ${ }^{1}$, \\ Ashish Misra', Elango Subramoniapillai ${ }^{1}$, Simon Durrant ${ }^{1}$ and Glen A. Kennedy ${ }^{1,2}$
}

Human leukocyte antigen (HLA) matched allogeneic stem cell transplant (SCT) is an established curative treatment for various haematologic malignancies ${ }^{1}$. However, a fully matched HLA-donor may still not be identified in a significant proportion of patients. Those who do not have an available HLA-matched donor can still potentially undertake transplantation utilizing an alternative donor source, including mismatched unrelated donors (MMUD), umbilical cord blood (UCB) or haploidentical donors $^{2}$. Although MMUD SCT is associated with increased risk of both graft versus host disease (GVHD) and non-relapse mortality (NRM), MMUD have still historically been utilized as alternative donor sources due to ease of donor availability ${ }^{3}$.

Historically, our Unit has offered MMUD SCT to individuals with high-risk haematological malignancies without an available HLA-matched donor. We aimed to review the outcome of consecutive patients undertaking MMUD SCT for acute leukaemia and myelodysplastic syndrome (MDS)/myeloproliferative neoplasm (MPN) performed at our institution between January 2004 and December 2014. Consent for release of information was obtained as per approved institutional practice for clinical audit. Conditioning protocols included myeloablative (cyclophosphamide $120 \mathrm{mg} / \mathrm{kg}$ and $12 \mathrm{~Gy}$ TBI or melphalan $140 \mathrm{mg} / \mathrm{m}^{2}$ and 12 Gy TBI) and reduced intensity conditioning (fludarabine $125 \mathrm{mg} / \mathrm{m}^{2}$ plus melphalan 120

\footnotetext{
Correspondence: Glen A Kennedy (glen.kennedy@health.qld.gov.au)

'Department of Bone Marrow Transplantation, Royal Brisbane and Women's Hospital, Brisbane 4029, Australia

${ }^{2}$ School of Medicine, University of Queensland, St Lucia, Queensland, Australia Full list of author information is available at the end of the article
}

$\mathrm{mg} / \mathrm{m}^{2}$ ). All grafts were T-replete, with no use of ATG/ Alemtuzumab, and peripheral blood progenitor cells (PBPC) used as stem cell source in all cases. GVHD prophylaxis consisted of cyclosporine and day 1, 36 and 11 methotrexate \pm administration of a single dose of Tocilizumab (TCZ) of $8 \mathrm{mg} / \mathrm{kg}$ at day- 1 of SCT as part of a prospective phase II study being conducted at the time. Prior to 2008, class I typing was performed using low resolution sequence specific primers (SSP), and high resolution sequence based typing (SBT) methods used only for HLA class II alleles. After 2008, SBT methods were utilized for both class I and II typing. Survival analysis was calculated from day of transplant to relapse or last follow up for progression free rate (PFR), and to death or last follow up for OS. NRM, acute GVHD incidence and GVHD-free, relapse-free survival (GRFS) ${ }^{4}$ were determined from the day of transplant. Survival analysis was performed using the Kaplain-Meier method. Acute and chronic GVHD were classified according to the Seattle criteria ${ }^{5,6}$.

In total 52 patients underwent MMUD SCT for AML/ MDS/MPN during the time period under review, representing $7.7 \%$ of all allogeneic SCT performed at our institution for these indications over this time. Baseline characteristics of are shown in Table 1 . In total, 40 donor/ recipient pairs $(77 \%)$ were mismatched at 1 loci only, including 23 with mismatches at HLA class I (44\%) and 17 $(33 \%)$ at class II loci. Mismatches at $>1$ loci occurred in 12 cases (23\%), including 7 cases involving $C$-mismatches $(\mathrm{C}+\mathrm{B}, n=2 ; \mathrm{C}+\mathrm{DR} \beta 1, n=2 ; \mathrm{C}+\mathrm{A}, n=1 ; \mathrm{C}+\mathrm{DQ}, n$ $=1 ; \mathrm{C}+\mathrm{B}+\mathrm{DQ}, n=1)$, and 5 cases involving $\mathrm{DQ}$ mismatches (DQ + DR $\beta 1, n=3$; DQ $+\mathrm{A}, n=1 ; \mathrm{DQ}+\mathrm{B}, n$ $=1$ ). Of the 12 multi-mismatched donor/recipient pairs, 
Table 1 Patient demographics and SCT details

\begin{tabular}{|c|c|}
\hline $\begin{array}{l}\text { Total } \\
\text { Age (median; range) }\end{array}$ & $\begin{array}{l}\mathrm{N}=52 \\
45 \text { yrs (range } \\
17-65 y r s)\end{array}$ \\
\hline \multicolumn{2}{|l|}{ Gender } \\
\hline Male & $23(44 \%)$ \\
\hline Female & $29(56 \%)$ \\
\hline \multicolumn{2}{|l|}{ Disease } \\
\hline AML & $31(59 \%)$ \\
\hline ALL & $16(31 \%)$ \\
\hline MDS/MPN & $5(10 \%)$ \\
\hline \multicolumn{2}{|l|}{ Risk category ${ }^{a}$} \\
\hline $\begin{array}{l}\text { Poor risk cytogenetics (including > CR1/2nd } \\
\text { AML) }\end{array}$ & $9(5)$ \\
\hline FLT3 ITD positive (including > CR1) & $4(1)$ \\
\hline $\begin{array}{l}>C R 1 \text { without poor risk cytogenetics } \\
\text { (including 2nd AML) }\end{array}$ & $8(3)$ \\
\hline $\begin{array}{l}\text { 2nd AML in CR1 (without poor risk } \\
\text { cytogenetics) }\end{array}$ & 10 \\
\hline \multicolumn{2}{|l|}{ ALL } \\
\hline $\begin{array}{l}\text { Poor risk cytogenetics (including high WCC/ } \\
\text { age }>35 \text { ) }\end{array}$ & $11(9)$ \\
\hline$>C R 1$ & 5 \\
\hline \multicolumn{2}{|l|}{ MDS/MPN } \\
\hline IPSS intermediate 2 or above & 5 \\
\hline \multicolumn{2}{|l|}{ Conditioning regimen } \\
\hline $\mathrm{Cy} / \mathrm{TBI}$ & $32(62 \%)$ \\
\hline $\mathrm{Mel} / \mathrm{TBl}$ & $1(1 \%)$ \\
\hline Flu/Mel & $19(37 \%)$ \\
\hline \multicolumn{2}{|l|}{ GVHD prophylaxis } \\
\hline MTX/CSA & $45(87 \%)$ \\
\hline MTX/CSA/TCZ & $7(13 \%)$ \\
\hline
\end{tabular}

Cy/TBI cyclophosphamide/TBI, Mel/TBI melphalan/TBI, Flu/Mel fludaraine+ melphalan

*Poor risk cytogenetics defined as per SWOG risk stratification for AML; ${ }^{11} t(9 ; 22)$, $t(4: 11)$, complex karyotype, low hypodiploidy/near triploidy, age $>35$ yrs, WCC $>$ $35 \times 10^{9} / \mathrm{l}$ for B-ALL and $>100 \times 10^{9} / \mathrm{l}$ for T-ALL for high risk ALL ${ }^{12-14}$, as per IPSS for $\mathrm{MDS} / \mathrm{MPN}^{15}$

class 1 HLA-mismatches were identified by low resolution typing in 8 of 9 cases with class 1 mismatches present. For the whole cohort, direction of mismatch was bidirectional in 49 cases (94\%) and in a "host versus graft" direction in three cases $(6 \%)$, including $\times 2$ single mismatched donor/ recipient pairs (single $\mathrm{C}+\mathrm{DQ}$ mismatches respectively) and $\times 1$ multi-mismatched donor/recipient pair $(B+C$ mismatched pair).
Neutrophil and platelet engraftment occurred in $98 \%$ and $94 \%$ of patients, respectively, at a median of 16 days (range 11-141 days) and 19 days (range 7-141 days) postSCT; 3 patients $(6 \%)$ died prior to platelet engraftment. All but one patient surviving past D28 post-SCT achieved full donor chimerism on short tandem repeats (STR) analysis of nucleated cells in peripheral blood. Overall incidence of grade II-IV and III-IV acute GVHD was 50\% and $17 \%$, respectively, with $84 \%$ of patients surviving post D100 $(n=37)$ developing chronic GVHD. Chronic GVHD was limited in 9 patients $(20 \%$ of patients surviving post D100) and extensive in 28 (64\% of surviving patients). Of patients with extensive stage chronic GVHD $>2$ organs were involved in $89 \%$, including liver in 10 cases (36\%) and lungs in 6 (21\%).

At median follow-up of survivors of 63mths (range 11-169mths) 5 yr OS, PFR and NRM and was 56\%, 53\% and $30 \%$, respectively (Fig. 1), with 12 month GRFS $29 \%$. To date 24 deaths have occurred, including 10 related to relapsed disease (42\%) and 14 (58\%) related to NRM. In total, 13 patients suffered relapsed disease at a median of $8 \mathrm{mths}$ post-SCT (range 2-74mths). Causes of NRM included infection $(n=6)$, GVHD $(n=4)$, secondary malignancies $(n=2)$, post-transplant microangiopathy $(n$ $=1)$ and failed engraftment $(n=1)$.

On univariate analysis, the only factor predictive of OS was development of grade III-IV GVHD ( 2 yr OS $71 \%$ versus $44 \%$ respectively, $p=0.009$ ). There was no association between $\mathrm{OS}$ and conditioning intensity, age $>$ versus $<$ median, degree of HLA mismatch or underlying disease. Specifically, no significant difference in OS was noted between HLA class 1 versus class 2 mismatched donors, or single versus multiple HLA-mismatched donors. Although there was no significant difference observed with respect to 12 month GRFS and degree of HLA mismatch, a favourable trend was noted with respect to both improved $12 \mathrm{~m}$ th GRFS with single versus multiple HLA mismatched donors (HR 1.66 (95\% CI 0.91-3.04); $P=0.098)$, as well as HLA class 1 versus class 2 mismatched donors (HR 1.45 (95\% CI 0.92-2.23); $p=0.11$ ). Treatment with TCZ did not appear to have any impact on reducing incidence of acute or chronic GVHD or $12 \mathrm{mth}$ GFRS.

Traditionally, MMUD transplants have been associated with increased rates of acute and chronic GVHD with corresponding poor OS related to high risk of $\mathrm{NRM}^{3,7}$. A majority of studies assessing outcomes post MMUD SCT are from large registries and have included patients receiving ATG as T-cell depletion and/or bone marrow grafts (as opposed to peripheral blood) as stem cell source $^{3,7,8}$. Our study differs from these in that we exclusively examined outcomes in consecutive patients undertaking T-replete MMUD SCT with PBSC grafts, i.e., a transplant platform associated with increased risk of 




Fig. 1 Survival outcomes for patient cohort. a OS, b PFR and c NRM. treatment and supportive care in GVHD over the last several years. The relatively low relapse rate realized in our cohort highlights the potential high curative potential of MMUD transplantation in high-risk malignancies, albeit at a cost of high risk of GVHD. Although patient numbers were low, administration of a single dose of TCZ at day -1 of SCT did not appear to have any impact on reducing incidence of acute or chronic GVHD or GFRS.

Previously published data have suggested that mismatch at HLA class 1 alleles is especially associated with significantly reduced OS when compared to matched unrelated donor transplants, and that presence 2 versus 1 HLA-mismatches are also associated with inferior outcomes $^{1,3,8-10}$. Overall, clinical impact of low versus high resolution HLA mismatches on OS, disease free survival, NRM or incidence of acute GVHD appears insignificant $^{3}$, and impact of individual HLA class 1 and class 2 mismatches on survival and GVHD outcomes remains controversial ${ }^{3,9}$. Likely related to the relatively low number of patients with specific HLA-mismatches within our cohort, we were unable to demonstrate any significant association between degree of HLA mismatch and GVHD incidence and/or OS.

MMUD remain a potentially important source of stem cells for patients with aggressive haematological conditions who do not have a readily available donor. However, despite the encouraging survival rates seen in our cohort, our experience confirms the high risk of both acute and chronic GVHD with use of MMUD. Transplant approaches that reduce GVHD incidence without increasing relapse rates are clearly required. Post-transplant high dose cyclophosphamide is potentially one such option and should continue to be examined as an alternative approach in this setting.

\section{Author details}

'Department of Bone Marrow Transplantation, Royal Brisbane and Women's Hospital, Brisbane 4029, Australia. ${ }^{2}$ School of Medicine, University of Queensland, St Lucia, Queensland, Australia. ${ }^{3} \mathrm{Q}$ IMR Berghofer Medical Research Institute, Brisbane, Queensland 4006, Australia

\section{Competing interests}

The authors declare that they have no competing interests.

\section{Publisher's note}

Springer Nature remains neutral with regard to jurisdictional claims in published maps and institutional affiliations.

Received: 19 June 2017 Revised: 20 August 2017 Accepted: 23 August 2017 Published online: 15 December 2017

\section{References}

1. Nakamae, H. et al. Low-dose total body irradiation and fludarabine conditioning for HLA-Class I mismatched donor stem cell transplantation and immunological recovery in patients with hematological malignancies: a multicenter trial. Biol. Blood. Marrow. Transplant. 16, 384 (2010). 
2. Ballen, K. K., Koreth, J., Chen, Y.-B., Dey, B. R. \& Spitzer, T. R. Selection of optimal alternative graft source: mismatched unrelated donor, umbilical cord blood, or haploidentical transplant. Blood. 119, 1972-1980 (2012).

3. Lee, S. J. et al. High-resolution donor-recipient HLA matching contributes to the success of unrelated donor marrow transplantation. Blood. 110 4576-4583 (2007).

4. Holtan, S. G. et al. Composite end point of graft-versus-host disease-free, relapse-free survival after allogeneic hematopoietic cell transplantation. Blood. 125, 1333-1338 (2015).

5. Glucksberg, $\mathrm{H}$. et al. Clinical manifestations of graft-versus-host disease in human recipients of marrow from HL-A-matched sibling donors. Transplantation. 18, 295-304 (1974).

6. Shulman, H. M. et al. Chronic graft-versus-host syndrome in man: a long-term clinicopathologic study of 20 Seattle patients. Am. J. Med. 69, 204-217 (1980).

7. Pidala, J. et al. Nonpermissive HLA-DPB1 mismatch increases mortality after myeloablative unrelated allogeneic hematopoietic cell transplantation. Blood. 124, 2596-2606 (2014)

8. Woolfrey, A. et al. HLA-C antigen mismatch is associated with worse outcome in unrelated donor peripheral blood stem cell transplantation. Biol. Blood. Marrow. Transplant. 17, 885-892 (2011).

9. Flomenberg, $\mathrm{N}$. et al. Impact of HLA class I and class || high-resolution matching on outcomes of unrelated donor bone marrow transplantation:
HLA-C mismatching is associated with a strong adverse effect on transplantation outcome. Blood. 104, 1923-1930 (2004).

10. Spellman S. et al. A standard definition of HLA matching for hematopoietic stem cell transplant outcomes studies. Biol Blood Marrow Transplant. 12(2; Suppl 1), 74 (abstr. 211). (2006).

11. Slovak, M. L. et al. Karyotypic analysis predicts outcome of preremission and postremission therapy in adult acute myeloid leukemia: a Southwest Oncology Group/Eastern Cooperative Oncology Group study. Blood. 96, 4075-4083 (2000).

12. Moorman, A. V. et al. Karyotype is an independent prognostic factor in adult acute lymphoblastic leukemia (ALL): analysis of cytogenetic data from patients treated on the Medical Research Council (MRC) UKALLXII/ Eastern Cooperative Oncology Group (ECOG) 2993 trial. Blood. 109 3189-3197 (2007).

13. Bassan, R. \& Hoelzer, D. Modern therapy of acute lymphoblastic leukemia. J. Clin. Oncol. 29, 532-543 (2011)

14. Ganzel, C. \& Rowe, J. M. Prognostic factors in adult acute leukemia. Hematol. Oncol. Clin. North. Am. 25, 1163-1187 (2011)

15. Greenberg, P. L. et al. Revised international prognostic scoring system for myelodysplastic syndromes. Blood. 120, 2454-2465 (2012). 